\title{
A bridge resistance deviation-to-time interval converter for resistive sensor bridges
}

\author{
Hoon Kim ${ }^{1}$, Won-Sup Chung ${ }^{2 a)}$, Sang-Hee Son ${ }^{2}$, and Hee-Jun Kim ${ }^{1}$ \\ ${ }^{1}$ School of Electrical Engineering and Computer Science, Hanyang University, \\ Ansan 425-791, Korea \\ ${ }^{2}$ Department of Semiconductor Engineering, Cheongju University, \\ Cheongju 360-764, Korea
}

a) circuit@cju.ac.kr

Abstract: A bridge resistance deviation-to-time interval converter is presented for interfacing resistive sensor bridges. It consists of two voltage-to-current converters, two current-tunable Schmitt triggers, a ramp integrator, and two logic gates. A prototype circuit built using discrete components exhibits a conversion sensitivity amounting to $4527.8 \mu \mathrm{s} / \Omega$ over the resistance deviation range of $0-2 \Omega$ and a linearity error less than $\pm 0.01 \%$.

Keywords: resistive bridge sensor, resistive bridge circuit, bridge resistance deviation-to-time interval converter

Classification: Integrated circuits

\section{References}

[1] C. D. Johnson and H. A. Richeh, "Highly accurate resistance deviation to frequency converter with programmable sensitivity and resolution," IEEE Trans. Instrum. Meas., vol. 35, pp. 178-181, 1986.

[2] K. Mochizuki and K. Watanabe, "A high resolution, linear resistancetofrequency converter," IEEE Trans. Instrum. Meas., vol. 45, pp. 761-764, 1996.

[3] P. A. Passeraub, P.-A. Besse, C. de Raad, and R. S. Popovic, "A differential relaxation oscillator as a versatile electronic interface for sensors," Sens. Actuators A, vol. 58, pp. 141-148, 1997.

[4] V. Ferrari, D. Marioli, and A. Taroni, "Oscillator-based interface for measurand-plus-temperature readout from resistive bridge sensors," IEEE Trans. Instrum. Meas., vol. 49, pp. 585-590, 2000.

[5] W.-S. Chung and K. Watanabe, "A temperature difference-to frequency converter using resistance temperature detectors," IEEE Trans. Instrum. Meas., vol. 39, pp. 676-677, 1990.

[6] C. B. Morlan, B. O. Buafull, G. M. Miranda, and A. Regueiro-Gomez, "A low cost circuit with direct digital output for pressure measurement," IEEE Trans. Instrum. Meas., vol. 48, pp. 817-819, 1999. 


\section{Introduction}

Due to their high sensitivity, resistive sensor bridges with four sensors are widely employed in industrial and process control systems, and medical instrumentation. A typical way of converting the resistance deviation into a digital form is to use a relaxation oscillator whose frequency is determined by the resistance under measurement and a known capacitor $[1,2,3,4]$. The use of a sinusoidal oscillator is also possible and reported in [5]. The other method for obtaining a digital output is to convert the resistance deviation into a time interval. A bridge resistance deviation-to-time interval (BRD-toTI) converter based on pulse-width modulators and digital frequency differentiation has been reported in [6]. This converter allows accurate and linear conversion of resistance deviation over time. However, unfortunately, this linear conversion is allowed for only small resistance deviation. This arises from the nonlinear (logarithmic) relationship between the input voltage and the output pulse width of the pulse-width modulator. The need of an external clock for triggering the modulators is another disadvantage of the converter.

In this paper a new BRD-to-TI converter based on current-tunable Schmitt triggers and digital frequency differentiation is presented. The proposed converter does not suffer from the above mentioned disadvantages, and thus it allows BRD-to-TI conversion with high linearity and sensitivity. This property makes it especially suit for detecting a small resistance variation with large fixed offset resistance.

\section{Circuit Description and Operation}

Figure 1 shows the circuit diagram of the bridge resistance deviation-to-time interval (BRD-to-TI) converter. It consists of a resistive sensor bridge with four sensors, two voltage-to-current (V-I) converters, a ramp integrator, two current-tunable Schmitt triggers, and two logic gates. The resistive sensor bridge provides the voltage outputs given by

$$
\begin{aligned}
& V_{1}=\frac{V_{C C}}{2}\left(1-\frac{\Delta R}{R}\right) \\
& V_{2}=\frac{V_{C C}}{2}\left(1+\frac{\Delta R}{R}\right)
\end{aligned}
$$

where $V_{C C}$ is the dc source exciting the bridge and $\Delta R$ represents the change in resistance of the sensors. $V_{1}$ and $V_{2}$ are converted into the currents $I_{B 1}$ and $I_{B 2}$

$$
\begin{aligned}
& I_{B 1}=\frac{1}{R_{1}}\left(V_{R}-\frac{V_{C C}}{2}-\frac{\Delta R}{2 R} V_{C C}\right) \\
& I_{B 2}=\frac{1}{R_{1}}\left(V_{R}-\frac{V_{C C}}{2}+\frac{\Delta R}{2 R} V_{C C}\right)
\end{aligned}
$$

respectively, by the V-I converters consisting of an operational amplifier, a $p n p$ transistor, a resistor $R_{1}$, and a reference voltage $V_{R} . I_{B 1}$ and $I_{B 2}$ control the threshold voltages of the current-tunable Schmitt triggers, which are composed of a voltage comparator, an operational transconductance amplifier 


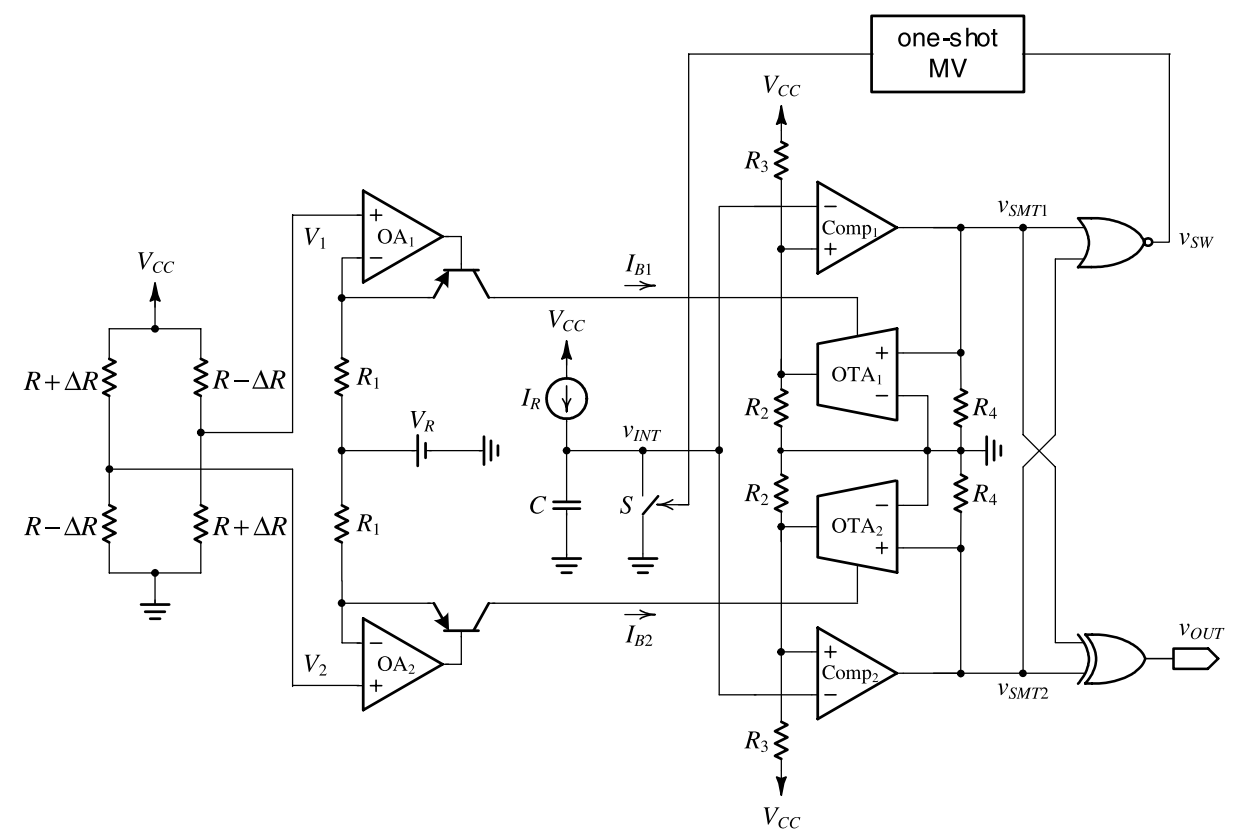

Fig. 1. Circuit diagram of the proposed bridge resistance deviation-to-time interval converter.

(OTA), and three resistors $\left(R_{2}, R_{3}\right.$, and $\left.R_{4}\right)$. The threshold voltages of the schmitt triggers are expressed as

$$
\begin{aligned}
V_{T L} & =\frac{R_{2}}{R_{2}+R_{3}} V_{C C} \\
V_{T H 1} & =V_{T L}+\left(R_{2} / / R_{3}\right) I_{B 1} \\
V_{T H 2} & =V_{T L}+\left(R_{2} / / R_{3}\right) I_{B 2}
\end{aligned}
$$

where $V_{T L}$ denotes the low threshold voltages of the Schmitt triggers, $V_{T H 1}$ denotes the high threshold voltage of the upper Schmitt trigger, and $V_{T H 2}$ denotes the high threshold voltage of the lower Schmitt trigger. Note that $V_{T H 1}$ and $V_{T H 2}$ are proportional to the current $I_{B 1}$ and $I_{B 2}$, respectively.

To see how the BRD-to-TI converter operates, refer to Fig. 2 which shows the signal waveforms at the various nodes of the converter, and assume that both of the Schmitt triggers are at their positive saturation level $V_{C C}$ and the bridge is unbalanced. Prior to the start of the conversion cycle, the switch $S$ connected in the ramp integrator is closed, thus discharging the timing capacitor $C$ of the ramp integrator and setting the input voltages of the Schmitt triggers $v_{I N T}$ to $0 \mathrm{~V}$. The conversion cycle begins with opening the switch $S$. Since the reference current $I_{R}$ flows through the capacitor, $v_{I N T}$ rises linearly with a slope of $I_{R} / C$. When $v_{I N T}$ reaches the high threshold voltage of the upper Schmitt trigger $V_{T H 1}$, the output of the upper Schmitt trigger $v_{S M T 1}$ falls to zero and the output of the XOR gate $v_{O U T}$ becomes high. Denoting $T_{1}$ the time duration for which $v_{S M T 1}$ keeps $V_{C C}$, we can write

$$
T_{1}=\frac{C}{I_{R}} V_{T H 1}=\frac{C}{I_{R}}\left[V_{T L}+\left(R_{2} / / R_{3}\right) I_{B 1}\right] .
$$

The conversion process continues until $v_{I N T}$ reaches the high threshold voltage of the lower Schmitt trigger $V_{T H 2}$. At this instant the output of the 


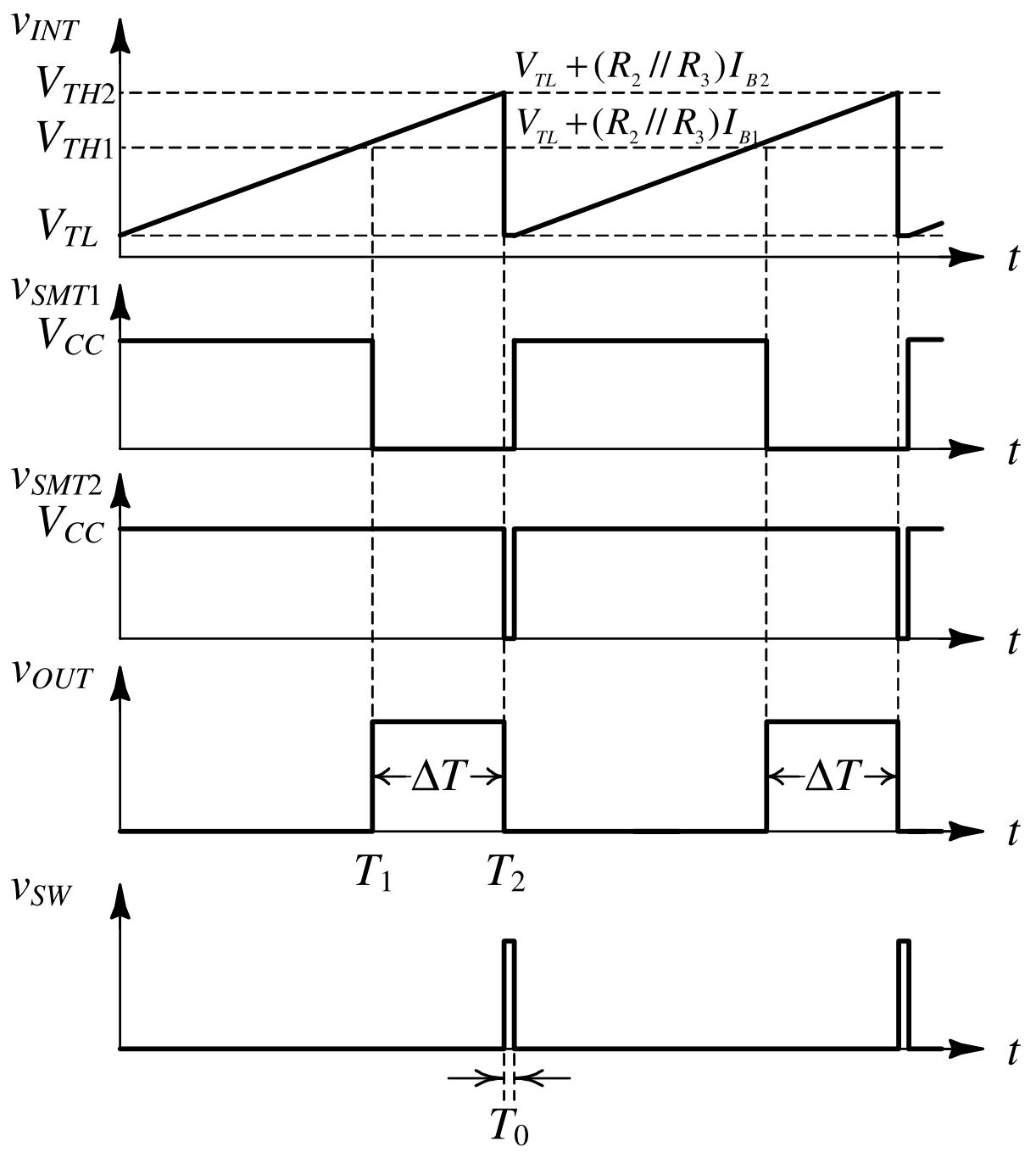

Fig. 2. Voltage waveforms at the various nodes of the converter.

lower Schmitt trigger $v_{S M T 2}$ falls to zero, thereby $v_{O U T}$ becomes low and the output of NOR gate $v_{S W}$ becomes high. The switch $S$ is now closed and thus clamping the voltage $v_{I N T}$ to ground. This in turn triggers the Schmitt triggers, causing their outputs rise to $V_{C C}$ and $v_{S W}$ go to low. The switch $S$ is now opened after the fixed duration $T_{0}$ of the one-shot multivibrator and a new conversion process is started. Denoting $T_{2}$ the time duration for which $v_{S M T 2}$ keeps $V_{C C}$, we can write

$$
T_{2}=\frac{C}{I_{R}} V_{T H 2}=\frac{C}{I_{R}}\left[V_{T L}+\left(R_{2} / / R_{3}\right) I_{B 2}\right] .
$$

The time interval of $v_{\text {OUT }}$ pulse is given by

$$
\Delta T=T_{2}-T_{1}=\frac{C\left(R_{2} / / R_{3}\right)}{I_{R}}\left(I_{B 2}-I_{B 1}\right) .
$$

Combining (2) and (6), one can obtain

$$
\Delta T=\frac{C\left(R_{2} / / R_{3}\right) V_{C C}}{I_{R} R R_{1}} \Delta R .
$$


A digital equivalent output can be obtained by counting the time interval with an external clock. Equation (7) also indicates that the sensitivity and the resolution of the converter can be controlled by adjusting the dc current $I_{R}$.

\section{Experiment Results}

A prototype converter shown in Fig. 1 was breadboarded using the following discrete components: LM324 for the operational amplifiers, MPQ2907 for the transistors, MC14066 for the switch, LM339 for the comparators, LM13600 for the OTAs, 74LS02 for the NOR gate, 74LS86 for the XOR gate, and NE555 for the one-shot multivibrator. The passive component values were adopted as follow: $C=2 \mu \mathrm{F}, R_{1}=1 \mathrm{k} \Omega, R_{2}=3.3 \mathrm{k} \Omega, R_{3}=30 \mathrm{k} \Omega$, and $R_{4}=10 \mathrm{k} \Omega$. A Wilson current mirror and a resistor of $205 \mathrm{k} \Omega$ were used for producing the dc current $I_{R}$ of $17.6 \mu \mathrm{A}$. The supply voltage $V_{C C}$ was $+5 \mathrm{~V}$ and the reference voltage $V_{R}$ in the V-I converters was set to $+3 \mathrm{~V}$.

One arm of the bridge was constructed with a resistor of $300 \Omega$ in series with a potentiometer of $100 \Omega$. All resistors are of $0.5 \%$ tolerance. Figure 3 shows the measured time interval changes when $\Delta R$ was changed in $0.1 \Omega$ steps from its fixed offset value of $R=350 \Omega$. Resistance was measured using the Agilent digital multimeter type 34405A, and the HP frequency counter 53131A with a resolution of $0.01 \mu \mathrm{s}$ in "period mode" was used to measure the time interval of the output pulses. Figure 3 indicates that the offset error is $16.4 \mu \mathrm{s}$ with a conversion sensitivity amounting to $4527.8 \mu \mathrm{s} / \Omega$

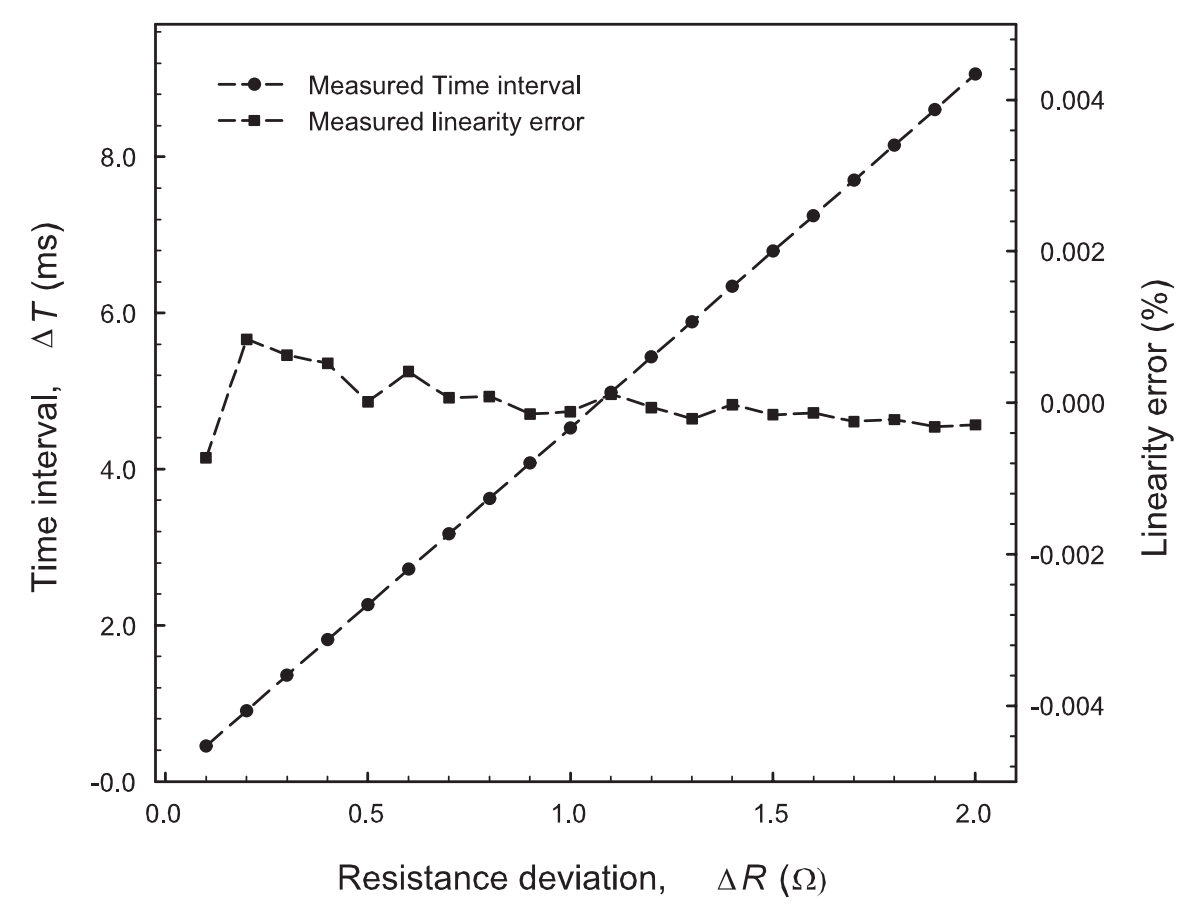

Fig. 3. Measured time interval versus resistance deviation and its linearity error. 
over the resistance deviation range of $0-2 \Omega$. This sensitivity is about 5520 times higher than that of the converter in [6]. The offset error is due to the mismatches of off-the-shelf components. The linearity error of the conversion characteristic is less than $\pm 0.01 \%$, which is adequate for most applications. The time interval drift of the converter itself was also measured by operating it for a few hours at a constant temperature. The measured drift was about $\pm 4 \mu \mathrm{s}$. Thus the minimum detectable resistance deviation of the converter is estimated to be $\pm 0.88 \mathrm{~m} \Omega$. The higher resolution can be obtained by adopting high-quality comparators.

\section{Conclusions}

A new circuit has been described which converts a resistance change in the bridge into its equivalent time interval change. The design principle and the circuit configuration are simple. Besides these, the converter features a high resolution and a good linearity even for a small change of the resistance. These properties make the converter suit for the signal processing of standard load cells.

\section{Acknowledgement}

This work was supported by the Korea Research Foundation Grant funded by the Korean Government (MOEHRD) (The Regional Research Universities Program/Chungbuk BIT Research-Oriented University Consortium). This work was also supported by IDEC in Korea. 